\title{
Proposta Brasileira de Metadados para Objetos de Aprendizagem Baseados em Agentes (OBAA)
}

\author{
Rosa Maria Vicari ${ }^{1}$, Marta Bez ${ }^{1}$, Júlia Marques Carvalho da Silva ${ }^{1}$, Alexandre Ribeiro ${ }^{1}$, João \\ Carlos Gluz ${ }^{2}$, Liliana Passerino ${ }^{1}$, Elder Santos ${ }^{1}$, Tiago Primo ${ }^{1}$, Luiz Rossi ${ }^{1}$, Alexandro \\ Bordignon ${ }^{1}$, Patricia Behar ${ }^{1}$, Raymundo Filho ${ }^{1}$, Valter Roesler ${ }^{1}$ \\ Universidade Federal do Rio Grande do SUL \\ Porto Alegre - RS - Brasil \\ rosa@inf.ufrgs.br,martabez@feevale.br; julia@univali.br; aribeiro@ucs.br; jcgluz@unisinos.br, \\ liliana@cinted.ufrgs.br,ersantos@inf.ufrgs.br,ttprimo@inf.ufrgs.br,lh.rossi@gmail.com, \\ alebordig@gmail.com,pbehar@terra.com.br,paka@ufrgs.br, roesler@inf.ufrgs.br
}

\begin{abstract}
This paper presents the Agent Based Learning Objects - OBAA standard proposal. The main goal of the research was to establish a standardized specification of the technical and functional requirements of learning objects that should allow the interoperability of the contents between Web and Digital TV environment, supporting accessibility and pedagogical issues. In this proposal it has been explored the convergence among the multiagent systems, $\mathrm{LO}$ and ubiquitous computing technologies, allowing the authoring, storage and recovery of LO in varied contexts and through different digital platforms. The result of this research was the definition of the OBAA proposal containing the requirements, specifications and architectures that will compose the Brazilian standard for the management, transmission, storage, search, editing and use of LO that can be distributed and used in an integrated manner within the Web platforms, mobile devices and Digital TV.
\end{abstract}

Palavras chave: Learning Objects, Agents, Metadata Standard, OBAA.

\section{Introdução}

O Learning Technology Standards Committee (IEEE LTSC, 2010) define que um objeto de aprendizagem é "qualquer entidade, digital ou não, que possa ser usada, reutilizada ou referenciada em um processo de aprendizagem”. Tais objetos de aprendizagem necessitam de metadados a fim de serem indexados, recuperados e reutilizados em repositórios (sistemas de gerenciamento de conteúdos). Os metadados de objetos de aprendizagem consistem em um arquivo que descreve o conteúdo educacional em aspectos técnicos e educacionais. Wiley (2001) descreve que os metadados são formados por um conjunto de informações para descrever um recurso com estrutura padronizada, facilitando a recuperação e acesso aos objetos de aprendizagem, ou em suas palavras: dados sobre dados.

Dentre as diversas organizações internacionais que criaram padrões para metadados educacionais, destacam-se o LOM (Learning Objects Metadata) do Learning Technology Standart Commitee do Institute of Electrical and Eletronic Engineers (IEEE/LTSC); o IMS Metadata do Instructional Management System (IMS) Global Consortium; e o Dublin Core Metadata Initiative.

No Brasil, a primeira iniciativa na busca de um padrão para objetos de aprendizagem foi o projeto Objetos de Aprendizagem Baseados em Agentes (OBAA), apresentado neste artigo. Este foi desenvolvido pela Universidade Federal do Rio Grande do Sul (UFRGS) em parceria com a Universidade do Vale dos Sinos (UNISINOS). A proposta foi uma resposta a chamada dos Ministérios da Educação, Comunicação e Ciência e Tecnologia buscando projetos para lidar com questões de interoperabilidade de conteúdos digitais em diversas plataformas. A principal ideia deste projeto foi o estabelecimento de uma especificação 
padronizada para os requisitos técnicos e funcionais de uma plataforma de produção, edição e distribuição de conteúdos digitais interativos, nas plataformas Web, em dispositivos móveis e na televisão digital (Vicari et al, 2009).

A base do padrão OBAA é o padrão LOM, com todas as suas categorias e mais alguns metadados, complementando as categorias técnica e educacional e duas categorias novas relativas a aspectos de acessibilidade e segmentação.

Neste artigo é apresentado o projeto OBAA, a proposta de especificação de metadados para objetos de aprendizagem. O texto está organizado da seguinte forma: a proposta de padrão de metadados para objetos de aprendizagem baseados em agentes é apresentada na seção 2; na 3 são apresentados os principais perfis de metadados existentes no padrão; a seção 4 contempla alguns estudos de caso de objetos de aprendizagem interoperáveis e, em seguida, são apresentadas as conclusões deste trabalho.

\section{Metadados}

Vários autores buscam metáforas para identificar os metadados, como Silva (2004), que faz uma comparação dos metadados com etiquetas identificadoras do conteúdo de um objeto de aprendizagem, descrevendo como, onde e por quem foram desenvolvidos, para qual segmento são destinados, seu tamanho, aplicação e outras informações relevantes. De-Marchi e Costa (2003) comparam com um catálogo de biblioteca, fornecendo informações sobre um determinado recurso, permitindo, com isso a interoperabilidade, identificação, compartilhamento, integração, utilização, reutilização, gerenciamento e recuperação dos mesmos de maneira mais eficiente.

Vicari et al (2009) enfatizam que para que os objetos de aprendizagem sejam armazenados em repositórios e reutilizados em diversos ambientes de aprendizagem, é necessária a descrição de seu conteúdo de uma forma padronizada, o que permitirá o intercâmbio de informações.

Atualmente, existem duas especificações de metadados para objetos de aprendizagem aceitos mundialmente: o IEEE LOM (mantido pelo IEEE) e o Dublin Core (mantido pelo Dublin Core Metadata Initiative). O IEEE LOM é considerado um modelo completo, pois apresenta a um conjunto de metadados organizado em nove categorias. Contudo, também é considerado de difícil preenchimento devido a sua extensão. O Dublin Core tem apenas 15 elementos, que inclui os mais utilizados pelos professores e designers instrucionais. Ainda, ele pode ser usado por qualquer recurso digital, e não apenas por objetos de aprendizagem.

\section{OBAA e suas especificações}

A proposta de um novo padrão para a descrição de objetos de aprendizagem interoperáveis para ambientes educativos surgiu a partir da realidade de uso cada vez mais constante da Web e da perspectiva que isso se estenda à TV-Digital e aos dispositivos móveis.

Para a definição deste padrão (OBAA) foram estudadas as especificações de metadados internacionalmente reconhecidos para a descrição de conteúdos educacionais IEEE LOM (2002) e o IMS (2002), além das especificações para catalogação de arquivos multimídia e TV-Digital (2001), MPEG-7 (2004) e SBTVD (2008).

A opção por estender o padrão a especificação IEEE LOM surgiu da ampla aceitação que esta já obteve no meio acadêmico e a fácil adequação do seu conjunto de metadados, permitindo a inserção de novas categorias e elementos em categorias já existentes. A Figura 1 ilustra as categoriais em que os metadados foram agrupados. Os novos elementos visam atender às necessidades brasileiras em termos de tecnologia, educação, acessibilidade e 
segmentação. Na sequência são descritas categorias de metadados da especificação OBAA, que se diferenciam do padrão IEEE LOM, que foram modificadas ou criadas.

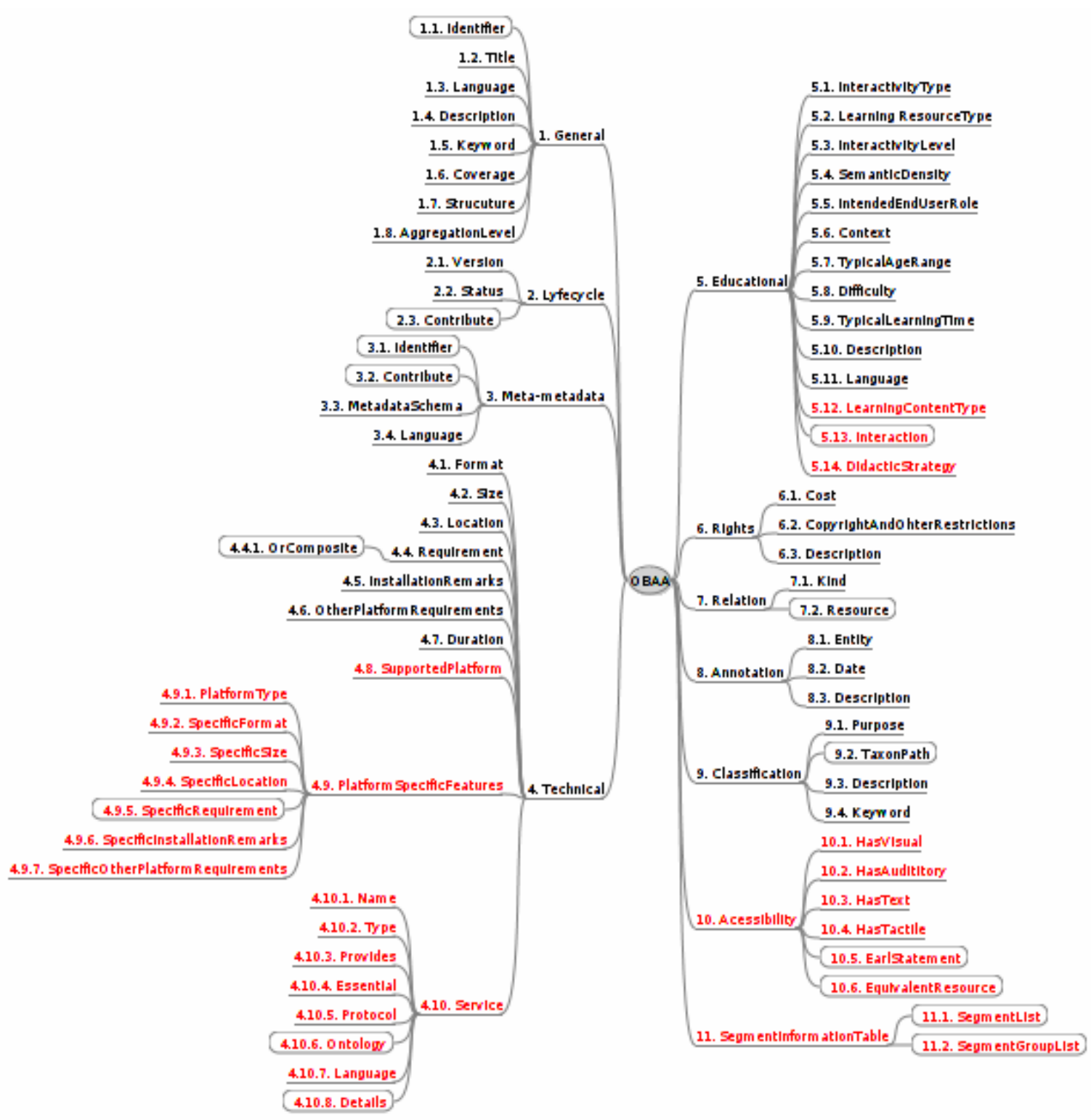

Fig. 1. Categorias dos metadados OBAA, com as extensões propostas com relação ao LOM em destaque

\subsection{Metadados Técnicos}

Como dito anteriormente, a base da categoria Technical do padrão LOM IEEE 1484 foi mantida, estendendo-a principalmente para dar suporte à interoperabilidade de plataformas. Nesta categoria foram definidas as informações técnicas e requisitos para utilização de objetos de aprendizagem com informações de mídia específicas para cada plataforma, ou seja, Web, TV Digital, Móveis.

Visando tratar de questões de interoperabilidade na Web semântica Berners Lee et al (2001), foram criados elementos para definir que serviços, ontologias, linguagens de conteúdo e protocolos de interação estão associados ao objeto. Estes são apresentados a seguir: 
SupportedPlatforms (4.8): prevendo os três tipos básicos de plataformas digitais para disponibilização de Objetos de Aprendizagem: Web, DTV e Mobile;

PlatformSpecificFeatures (4.9): os dados técnicos das mídias aplicados a cada uma das plataformas, para as quais o OA foi previsto são aqui identificados. Se uma plataforma for informada no item SupportedPlatforms e não for criado um grupo PlatformSpecificFeatures específico para essa, então os dados técnicos gerais, da categoria Technical do LOM, se aplicam também para a plataforma. Para cada plataforma que essa categoria de metadados for criada, deverão existir também informações do tipo de plataforma digital à qual se aplicam os parâmetros, o formato, o tamanho da mídia, forma de acesso, capacidades técnicas necessárias, instruções de instalação e requisitos de software;

Service (4.10): um conjunto de metadados é responsável pela associação aos seus respectivos serviços, podendo ser expandido para qualquer provedor de serviço disponível. Aqui a especificação dos metadados modela o acesso ao serviço, dando flexibilidade para que sejam utilizados os serviços necessários, independente da tecnologia utilizada, não apresentando restrições para a forma como ele pode ser implementado. Para tanto, são especificados itens como nome, tipo, definição do serviço, protocolo, ontologia, linguagem e detalhes. Essa definição do serviço pode ser obrigatória ou opcional, buscando a correta execução do objeto.

Os elementos SupportedPlatforms, PlatformSpecificFeatures e Service, seguem as mesmas definições e regras da categoria 4 do padrão LOM, porém, aplicadas a cada plataforma utilizada.

\subsection{Metadados Educacionais}

A categoria Educational também recebeu uma extensão, ampliando a quantidade de elementos desta categoria, tendo como base o modelo interacionista, partindo-se da premissa que o sujeito conhece o mundo por meio da interação com os objetos de conhecimento. Os seguintes aspectos foram levados em consideração para atender este modelo: organizacionais, referentes ao conteúdo, metodológicos e tecnológicos. O modelo pedagógico foi composto por uma arquitetura pedagógica e por uma concepção pedagógica e epistemológica do professor.

Foi criada a definição do elemento LearningContentType para representar este modelo pedagógico, especificando o tipo de conteúdo do objeto de aprendizagem. Este pode ser classificado em conteúdos factuais, conteúdos referentes a conceitos, conteúdos procedimentais, conteúdos atitudinais. [11]

A interação entre o objeto de aprendizagem e o usuário diz respeito ao comportamento das pessoas em relação a outras pessoas e sistemas. Parte-se do princípio que somente é possível proporcionar experiências de aprendizagem significativas se a solução educacional for projetada interativa. É necessário disponibilizar uma interface que possibilite interação oferecendo atividades de aprendizagem que exijam do aluno interação com conteúdos, ferramentas e com outras pessoas. O metadado interaction foi criado para definir essa interação. Este é composto pelo mecanismo sensorial utilizado para transmitir a informação, pela forma de interação entre o usuário e o objeto, mecanismos para informar e utilizar a copresença de outros usuários no ambiente e pela forma de relacionamento entre os usuários, necessária para o funcionamento deste objeto de aprendizagem. Reciprocidade é um modo de se relacionar com os outros no qual todos têm as mesmas oportunidades e chances de participação e de interação no grupo. Espera-se que essa operação possa auxiliar os alunos na 
aprendizagem cooperativa e colaborativa, onde, através de trocas, é possível aprender e ensinar.

Uma estratégia didática é definida como um conjunto de ações planejadas e conduzidas pelo professor para promover o envolvimento e o comprometimento dos alunos com um conjunto maior de atividades. Na especificação do padrão é possível indicar a estratégia didática mais adequada a ser adotada na utilização do objeto de aprendizagem, conforme a concepção do autor.

\subsection{Metadados de Acessibilidade}

Cada vez mais percebe-se a importância do tema acessibilidade. Para lidar com questões referentes a acessibilidade, foi criada uma nova categoria, não existente no LOM, denominada Acessibility. A base dos elementos desta categoria é o AccessForAll do IMS (2002). A intenção é armazenar informações de acessibilidade sobre o aluno, definindo as configurações dos usuários ao acessar o objeto de aprendizagem, permitindo atender requisitos como de áudio para cegos, legenda para surdos, idiomas e outras especificações importantes buscando fornecer acesso e inclusão ao objeto de aprendizagem às pessoas com necessidades educativas especiais. Neta categoria de metadados é descrita a necessidade para utilização dos recursos, se o visor e método de controle podem ser transformados e se existe uma alternativa equivalente conhecida. É Importante observar as subcategorias existentes aqui, como a especificação de conteúdos com informação visual, audível, textual e táctil.

Também foram contempladas referências à linguagem para Avaliação e Relato definida pelo W3C (2008) para expressar e comparar testes e resultados, transformabilidade e controle e flexibilidade dos recursos referenciados, também foram contempladas.

A possibilidade de apontar para um recurso equivalente ao recurso descrito ou partes do mesmo foi contemplada. Neste mesmo item, é possível a indicação de facilidades de acesso ao aprendizado que estão ou serão contidos no OA, como uma ou mais ferramentas de apoio. Alguns valores possíveis para este metadado são os descritos a seguir: dictionary, calculator, noteTaking, peerInteraction, abacus, thesaurus, spellChecker, homophoneChecker, mindMappingSoftware, outlineTool.

No caso da especificação de uma alternativa visual, pode ser definido se esta será em áudio, texto alternativo longo na linguagem especificada para o principal recurso referenciado, e descrição de como as cores devem ser utilizadas. Alguns exemplos de valores possíveis deste vocabulário são: avoidRed, avoidRedGreen, avoidBlueYellow, avoidGreenYellow, avoidOrange, avoidRedBlack, avoidPurpleGray, useMaximumContrast e Monochrome. É possível também definir se o recurso descrito contém alternativas gráficas/visuais para textos no texto principal, como a indicação de conteúdos traduzidos para linguagem de sinais, no dialeto específico sendo fiel ao conteúdo principal do recurso. Neste caso, alguns exemplos de valores possíveis são: American-ASL, British-BSL, Brazilian-BRA, Native Guarani-GUA, Spanish-SPA, French-LSF, Japanese-JSL, other.

\subsection{Metadados de Segmentação}

Às vezes, torna-se necessário segmentar logicamente um objeto, permitindo a organização do mesmo em módulos ou por assuntos tratados. Um segmento é um fragmento contínuo de um objeto. Um segmento particular pode pertencer a um único programa, mas pode ser membro de vários grupos de segmentos, dentando, neste caso, uma coleção de segmentos que são associados por uma finalidade particular ou devido a uma propriedade em comum. 
Para atender às necessidades de segmentação do padrão proposto foi adaptado o TVAnytime (2003), padrão de metadados utilizado pela TV Digital na Europa, simplificado e adaptado. Com isso foi criada uma nova categoria, denominada SegmentInformationTable, que contém os metadados de segmentação definidos pelo padrão MPEG-7.

A categoria 11 - segmentação dos objetos de aprendizagem (categoria 11) e de grupos de segmentos dos objetos de aprendizagem foi criada, esta contendo: identificadores, título, descrição, palavras-chave, tipo de segmento (documento texto, hiperdocumento, arquivo multimídia ou outros) e a indicação de início e fim do segmento no objeto de aprendizagem. Em cada segmento, são utilizadas as mesmas definições e regras de definição dos objetos de aprendizagem do padrão LOM, porém cada uma das partes do objeto.

\subsection{Sintaxe e Semântica dos Metadados}

A independência de tecnologia e flexibilidade da proposta do OBAA é garantida pela definição de sintaxe em XML e da semântica usando uma ontologia OWL (Web Ontology Language), compatível com a Web Semântica. A flexibilidade e ampla adoção pela comunidade científica originou a escolha desta linguagem. A modelagem definida para o OBAA está de acordo com a linguagem OWL-DL (Description Language), o que permite uma expressividade suficiente para a sua aplicação no contexto destes metadados, mas mantendo a completude computacional. Existe, por parte do grupo de desenvolvimento do OBAA, um interesse especial no uso conjunto da ontologia de metadados OBAA e nos perfis de metadados.

\section{Os Perfis de Metadados do OBAA}

Uma das características importantes do padrão IEEE LOM Std. 1484.12.1-2002, é o fato de não definir uma classificação única a respeito da obrigatoriedade, opcionalidade ou irrelevância de cada um dos metadados, podendo um metadado ser considerado opcional em um determinado contexto, e obrigatório em outro. Seguindo esta mesma linha de raciocínio, o padrão OBAA trabalha com Perfis de Metadados. Os Perfis de Metadados tem por objetivo a redução da complexidade no gerenciamento dos metadados, trabalhando com domínios, incluindo autoria, edição, armazenamento e inferência de informações sobre esses objetos. Os metadados dos objetos de aprendizagem formam uma biblioteca de metadados que podem ser usados para caracterizar as propriedades de um dado objeto de aprendizagem.

Um determinado objeto de aprendizagem estará de acordo com determinado perfil de metadados quando este contiver o conjunto mínimo de metadados especificados no perfil. Os metadados podem, em função do especificado, ser classificados como: obrigatórios (devem estar presentes para o objeto ser considerado valido no perfil) ou opcionais (puderem estar presentes ou não para que o objeto seja considerado válido no perfil).

Estes perfis não podem ser considerados substitutos para futuras ontologias mais completas, especificadas em OWL, que definiriam os elementos, estruturação e demais propriedades de domínios específicos de aplicação de objetos de aprendizagem, como, por exemplo, ontologias sobre determinados domínios do conhecimento. Estes visam servir de base para a criação e desenvolvimento de futuras ontologias, fornecendo dicionários de termos elementares e a estruturação inicial para essas ontologias. O conjunto de perfis definidos no OBAA é normativo, em outras palavras, futuras ontologias devem referenciar, no mínimo, qual perfil de metadados é usado como dicionário de termos. Os principais perfis de metadados (PM) que foram previstos no padrão OBAA são: 


\subsection{Perfil Adequação (PM-ADEQ-BASE)}

É composto por uma quantidade mínima de informações que devem ser guardadas a respeito de um determinado objeto. Este perfil deve ser usado é quando se conhece apenas o identificador único e seu endereço (URL) em um determinado repositório. Exemplo disso ocorre em situações em que os objetos legados ainda não foram analisados. Os metadados deste perfil serão o General.Identifier (1.1) e Technical.Location (4.3).

\subsection{Perfis de Aderência a Padrões de Metadados}

O objetivo deste perfil e' definir os requisitos de compatibilidade entre o padrão de metadados e outros padrões previamente definidos. Atualmente são previstos requisitos de compatibilidade funcional com o padrão DCMI não qualificado (RFC 5013) e compatibilidade estrita (strictly conforming) com o padrão IEEE-LOM Std. 1484.12.1-2002. O OBAA especifica um modelo abstrato OWL para seus metadados, incluindo os metadados IEEE-LOM nessa especificação. O objetivo de compatibilidade do perfil PM-DCMI é mapear, para fins de armazenamento, disponibilização e conversão, objetos de aprendizagem onde seus metadados tenham sido previamente especificados de acordo com o padrão DCMI em objetos de aprendizagem funcionalmente equivalentes, com conteúdos idênticos e com metadados já seguindo a presente proposta de padrão. Estes são divididos em:

a) Perfil Compatível com IEEE-LOM (PM-IEEE-LOM): tem por objetivo especificar os requisitos de conformidade estrita com o padrão IEEE-LOM Std. 1484.12.1-2002. Os metadados de um objeto de aprendizagem estarão estritamente conforme este padrão quando forem restritos aos metadados definidos na tabela 1 , seção 6 do LOM. O único metadado considerado obrigatório é o 1.1 General.Identifier, sendo os demais opcionais.

b) Perfil Compatível Mínimo com OBAA (PM-OBAA-CORE): é formado pelo conjunto de metadados mínimos considerados essenciais para todo objeto de aprendizagem descrito conforme o padrão OBAA. Busca-se neste perfil a integração com os objetos de aprendizagem legados, com reduzido esforço humano.

c) Perfil Compatível Ideal com OBAA (PM-OBAA-FULL): Consiste na quantidade ideal de informações que devem ser registradas sobre um objeto para que este contemple as diversas aplicações para TV Digital, dispositivos móveis e Web, garantindo conversões de conteúdo e interoperabilidade.

\subsection{Perfis de Aplicação}

Tem por objetivo definir subconjuntos de metadados aptos a tratar dos aspectos particulares de uma dada aplicação de objetos de aprendizagem, sendo divididos em:

a) Perfil Base para Aplicações Multiplataforma (PM-MULTI-BASE): define o conjunto mínimo de metadados necessários para suportar aplicações multimídia nas plataformas de disponibilização previstas nesta proposta de padrão.

b) Perfil Base para Aplicações Educacionais (PM-EDUC-BASE): define o conjunto mínimo de metadados necessários para suportar aplicações educacionais básicas. 
c) Perfil Base para Aplicações de Acessibilidade (PM-ACESS-BASE): define o conjunto mínimo de metadados necessários para aplicações com suporte a acessibilidades.

Estes perfis têm o objetivo de fornecer uma forma de redução da complexidade no gerenciamento dos metadados, quando restrita a certos domínios, tanto para fins de autoria, edição, armazenamento e inferência de informações sobre os objetos de aprendizagem. Espera-se a criação, definição e especificação de novos perfis, facilitando a disseminação e uso OBAA.

\section{Prova de conceitos - Objetos Interoperáveis}

Todo padrão, para se consolidar, necessita de provas de funcionamento. Visando comprovar os itens descritos no OBAA, foram adaptados e utilizados dois objetos de aprendizagem. $\mathrm{O}$ primeiro deles foi obtido do repositório SACCA, sendo seu conteúdo composto por um vídeo. O segundo objeto foi adquirido do NUTED, sendo seu conteúdo composto por imagens e páginas Web.

O primeiro objeto de aprendizagem utilizado é um vídeo que apresenta a origem da Televisão, contando um pouco de sua história. Também explica como é o funcionamento da transmissão de TV atualmente e apresenta também ao aluno um contexto amplo sobre o funcionamento dessa importante mídia. No formato original, esse vídeo se encontra no formato wmv e resolução 320x240, no repositório SACCA (Sistema Automático de Catalogação de Conteúdo Audiovisual).

Buscando a interoperabilidade, o vídeo foi convertido para o formato H.264/AAC LC e encapsulado num arquivo “.mp4”. Esse formato contempla a execução do vídeo no GingaNCL Virtual Set-top Box (para TV-Digital) e na Web. Também o vídeo também foi convertido para a codificação H.263/AAC LC, em formato de arquivo “.3gp”, para execução nos dispositivos móveis. Portanto, tem-se um vídeo em dois formatos distintos e que permitem a execução do conteúdo nos três ambientes pesquisados (Web, TV-Digital e móvel). A Figura 2 apresenta o vídeo nas três plataformas.
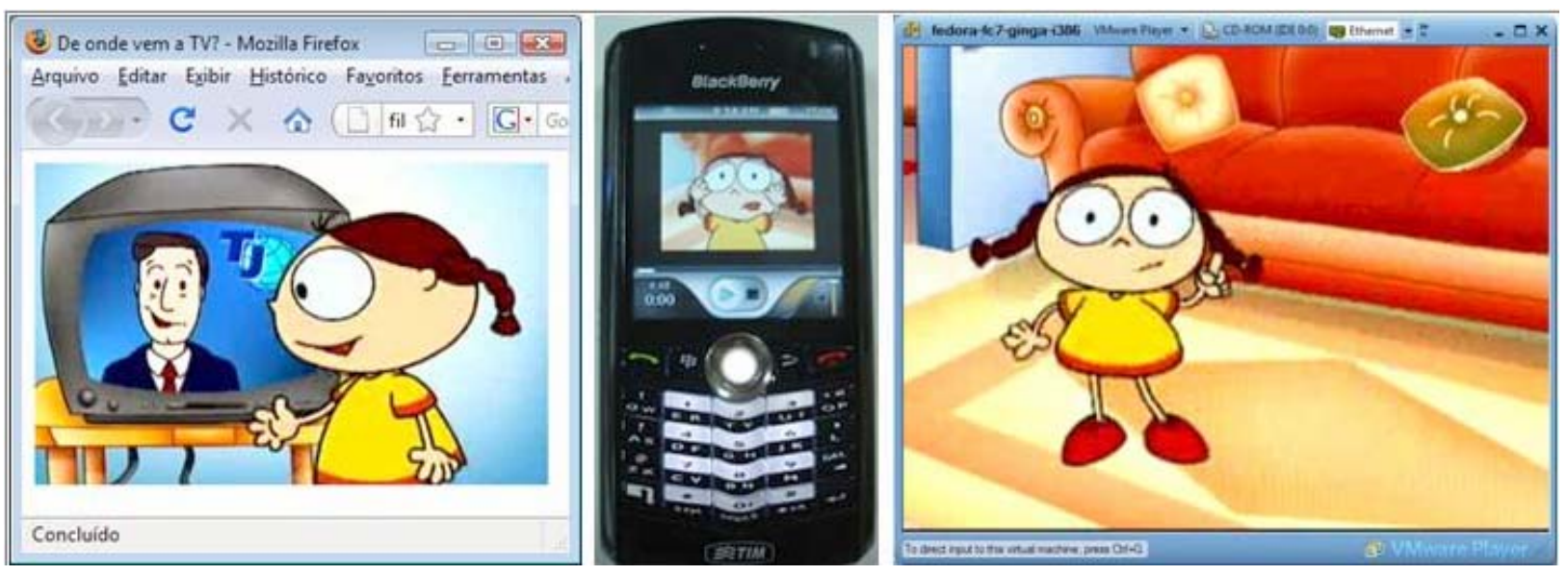

Fig. 2. Objeto de Aprendizagem “De Onde vem a TV?” nas três plataformas.

O segundo objeto trabalhado foi o objeto de aprendizagem “Outras Infâncias”. Este é um objeto de aprendizagem do NUTED (Núcleo de Tecnologia Digital Aplicada à Educação) da UFRGS, desenvolvido originalmente para a Web.

A versão original deste objeto de aprendizagem, de autoria do NUTED (Núcleo de Tecnologia Digital Aplicada a Educação - UFRGS), que possibilita o acesso via Web, está disponível em http://www.nuted.ufrgs.br/objetos/ei2007/infancias/index.html. 
Para o conteúdo texto utilizou-se o XDHTML e CSS para adaptar as imagens para exibição, seguindo-se as recomendações do OBAA. Na Figura 3 é apresentado o Objeto de Aprendizagem nas três plataformas, demonstrando a interoperabilidade do padrão.
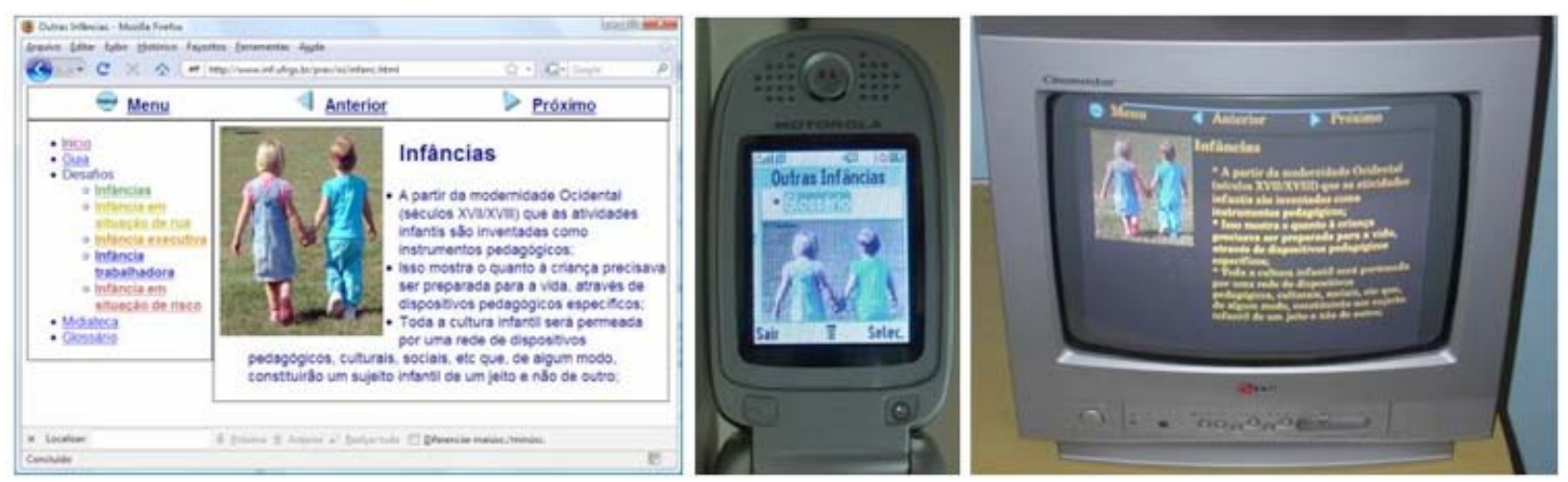

Fig. 3. Objeto de Aprendizagem “Outras Infâncias” nas três plataformas.

\section{Conclusão}

A busca da abertura tecnológica, o uso independente da plataforma gerou a proposta de especificação OBAA para objetos de aprendizagem interoperáveis na Web, TV-Digital e dispositivos móveis. Esta permite uma adaptação as plataformas existentes, bem como, flexibilidade para novas tecnologias.

Um padrão deve ser flexível e adaptável, o que exige um amplo estudo das particularidades de cada plataforma. Para contemplar as particularidades das três plataformas (Web, TV-Digital e dispositivos móveis) - heterogêneas entre si, em um único padrão que as compatibilize - é necessário lidar com os requisitos e complexidades de todas elas. Esta complexidade deve ser interna, provendo ao usuário a simplicidade. Promover essa compatibilização, atribuindo o máximo possível de liberdade para o desenvolvedor de conteúdo educacional, resultou em um amplo conjunto de metadados (nem sempre obrigatórios) e recomendações técnicas.

Espera-se que essa decisão de concepção permita uma grande aceitação do padrão OBAA pela comunidade da Educação e também que a mesma auxilie na evolução do padrão para atender às demandas da Educação no Brasil. Levou-se em consideração a existência de grande quantidade de objetos de aprendizagem já desenvolvidos, em diferentes padrões, que precisam ser adaptados.

Uma das formas para facilitar o uso do padrão é a definição de perfis de metadados (conjuntos reduzidos de metadados), direcionados aos desenvolvedores de conteúdo educacional. Através dos perfis é possível atender diferentes demandas de nichos específicos da comunidade educacional, mantendo a aderência ao padrão e reduzindo sua complexidade. No futuro, os perfis poderão ser usados para aplicar as respectivas definições para classificar automaticamente os objetos de aprendizagem segundo seu grau de interoperabilidade. Desta forma, é possível obter um serviço automatizado de classificação, que poderá ser usado por ferramentas de autoria e portais de conteúdo.

\section{Referências Bibliográficas}

Berners Lee, Tim; Hendler, James; Lassila, Ora. The Semantic Web. Scientific American 2001; 284(5), pg. 35-43. 
De-Marchi, Ana Carolina Bertoletti; Costa, Antônio Carlos da Rocha. Uma proposta de padrão de metadados para objetos de aprendizagem de museus de ciências e tecnologia. In: RENOTE - Revista Novas Tecnologias na Educação. Porto Alegre: Centro Interdisciplinar de Novas Tecnologias na Educação (CINTED - UFRGS), v. 1, n. 1, 2003. Disponível em: www.cinted.ufrgs.br/renote/mar2004/artigos/02-umapropostadepadrao.pdf. Acesso em 24/10/2009.

IEEE LTSC. Learning Technology Standards Committee. Disponível em:http://ltsc.ieee.org/wg12/ Acesso em maio de 2010.

IMS. IMS Global Learning Consortium. 2002. Inc. Disponível em: http://www.imsglobal.org/. Acesso em: 20/jun/2008.

ISO/IEC 15938-5. Information Technology - Multimedia content description interface - Part 5 Multimedia Description Schemes, 2001.

MPEG-7. MPEG-7 Overview (ISO/IEC JTC1/SC29/WG11 N6828). 2004. Disponível em: http://www.chiariglione.org/mpeg/standards/mpeg-7/mpeg-7.htm. Acesso em junho de 2008.

TELECO. Informação em Telecomunicações. 2008. Disponível em http://www.teleco.com.br. Acesso em 15 de outubro de 2008.

TVA. TV-Anytime Forum. S3 metadata (normative) v1.2. TV-Anytime Specification, 2003. Disponível em http://www.tv-anytime.org. Acesso em junho de 2008.

VICARI, R. et al. Padrão para Metadados de Objetos de Aprendizagem Multiplataforma. Porto Alegre: UFRGS, 2009.

W3C. Mobile Web Best Practices 1.0, Basic Guidelines. W3C Recommendation. 2008. Disponível em http://www.w3.org/TR/mobile-bp/. Acesso em 15 de junho de 2009.

WILEY, D. Connecting learning objects to instructional design theory: a definition, a metaphor, and taxonomy. 2001. Disponível em: www.reusability.org/read/chapters/wiley.doc. Acesso em: 07/08/2009.

SILVA, Maria da Graça Moreira da. Novas Aprendizagens. In: Congresso Internacional de Educação à Distância, São Paulo, 2004. Disponível em: www.abed.org.br/congresso2004/por/htm/146-TC-D2.htm. Acesso em 26/10/2009. 